\title{
Analytic formulas for the natural frequencies of hinged structures with taking into account the dead weight
}

\author{
Yurii Krutii ${ }^{1, *}$, Mykola Suriyaninov ${ }^{1}$, and Victor Vandynskyi ${ }^{1}$ \\ ${ }^{1}$ Odessa State Academy of Civil Engineering and Architecture, Didrikson str., 4, Odessa, 65029, \\ Ukraine
}

\begin{abstract}
Free bending vibrations of hinged vertical uniform rod with taking into account the dead weight are investigated. The research is based on the exact solution of the partial differential vibration equation with variable coefficients. This approach allows to get more reliable picture of rod's vibration because only the exact solution carries information of a qualitative nature and forms the most complete picture of the physical phenomenon under consideration. The frequencies equation of problem was written in dimensionless form and the way of its root finding is shown. It has been shown that the problem of determination the nature frequencies of structures is reduced to finding corresponding dimensionless vibration coefficients from equation. The formulas for the first three vibration frequencies of structures were obtained in analytical form. An analytic relationship between the frequencies with and without taking into account the dead weight of the structures was established. The nature of the dependence of frequencies on the value of the longitudinal load was revealed. The presence of conclusive analytical formulas for determining the vibration frequencies of hinged vertical structures with taking into account the dead weight is a real alternative for using the approximate methods for solving this class of problems of solid mechanics.
\end{abstract}

\section{Introduction}

When designing vertical structures, it is necessary to consider all possible impacts they may be exposed during construction and operation. Taking into account the fact, that recently the structures of high-rise building is intensively developing, including at the earthquakeprone areas, the relevance of investigation the different types of structures with dynamic load is undisputed.

The qualitative evaluation of the dynamic parameters of the relevant structures is required for the quality structural design. This evaluation can be obtained by analyzing such important characteristics as free vibration frequencies and mode shapes, obtained by investigation of the structure in the unloaded state. Based on these characteristics, we can

\footnotetext{
* Corresponding author: yurii.krutii@gmail.com
} 
make the conclusion about the rigidity and operability of the structure, correctness of design solutions.

Many materials and publications are concerned with research of different type of construction for bending vibrations with taking into account effect of longitudinal force.

In article [1] bending vibrations of uniform beams under various boundary conditions and axial loads have been studied. The paper [2] deals with the case when the axial force takes different constant values in different parts of the beam. Here, the first three vibration frequencies for various combinations of boundary conditions have been calculated. The authors of [3] study vibrations of frame structures taking into account the influence of axial load. The load is calculated based on the total weight and number of floors of the building. Natural frequencies of frame structures are obtained by solving the differential equation written for an equivalent rod whose rigidity and weight must be uniformly distributed along the length. In the paper [4] the necessity of taking into account the influence of an axial force on the free vibration frequencies of marine steel platform support blocks' grid elements during the design and reliability prediction of welded joints is proved. In the investigations of influences of load on bending vibration the axial load is often [5-7] accepted constant along the height for simplification. However, in the real structures the axial forces in different cross sections have different values.

One of the most widely used design schemes in structural design is the hinged vertical uniform rod under the variable longitudinal force, represented with dead weight of the structure.

The mathematical model of such a physical phenomenon is a differential variablecoefficient equation $[8,9]$. Its solution had been unknown until the work [10] published. Therefore, the studies were conducted by approximate, as a rule, variational methods [9]. However, the presence of an exact solution provides for qualitative evaluation of mechanical system. Based on the previously obtained results $[10,11]$, the objective of the work is to determinate in analytical form the formulas for the free vibration frequencies of vertical hinged structures with taking into account the dead weight; to establish in an analytical form the relationship between frequencies with taking into account the dead weight of the structures and without it.

\section{Results}

\subsection{General symbols and equations}

Let us analyze the free bending vibrations of the rod with uniform flexural rigidity and intensity of the distributed mass taking into account the longitudinal distribution force (Fig.1, Fig.2). Fig. 1 shows the general scheme of vibrations of the vertical hinged rod, and Fig. 2 shows the external and internal forces acting on its element. 


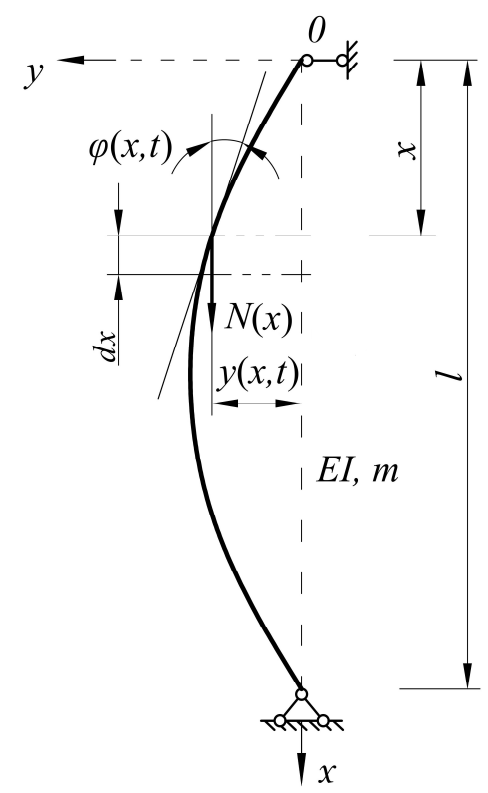

Fig. 1. The scheme of free bending vibrations of a straight rod

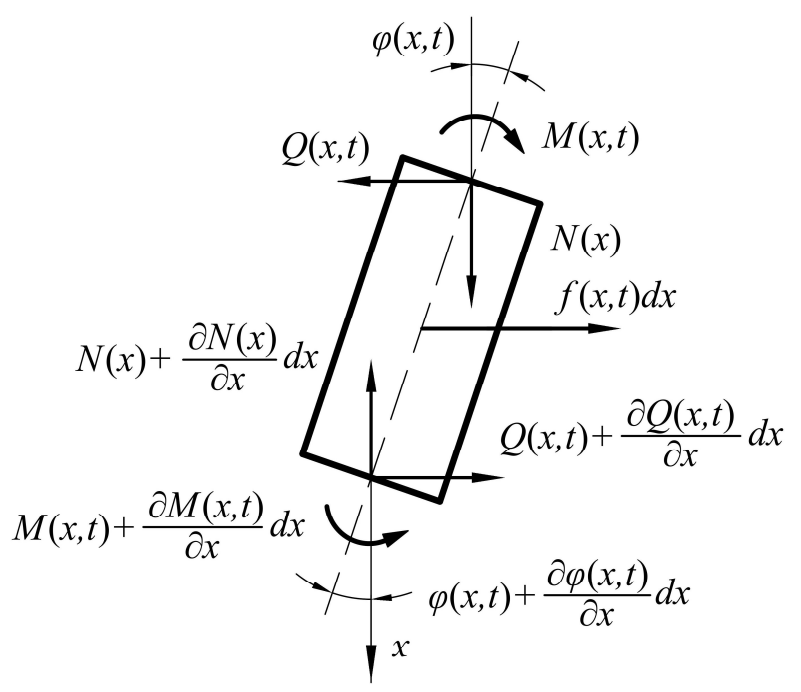

Fig. 2. The scheme of the forces acting upon the element of a rod

List of symbols:

$E I$ - flexural rigidity of a rod;

$m$ - intensity of the distributed mass (dead weight) of the rod;

$N(x)=q x-$ variable axial (compressive) force, where $q$ - weight per unit length of beam;

$y(x, t)-$ cross motion of the axis point of the rod with coordinate $x$ at time $t$ (dynamic deflection);

$\varphi(x, t)$ - dynamic angular displacement;

$M(x, t)$ - dynamic bending moment; 
$Q(x, t)$ - the dynamic transverse force;

$f(x, t)$ - the intensity of inertial forces that appear during vibration (D'Alembert force).

It is known [8,9], that the equation of free transverse vibrations account of own weight is written as

$$
E I \frac{\partial^{4} y}{\partial x^{4}}+q \frac{\partial}{\partial x}\left(x \frac{\partial y}{\partial x}\right)+m \frac{\partial^{2} y}{\partial t^{2}}=0 .
$$

In [10], the authors first found the exact solution of this differential equation. As a consequence, formulas were also obtained for the dynamic parameters of the state of the rod (deflection, angular displacement, bending moment and transverse force), which are expressed in terms of dimensionless fundamental solutions (1). The presence of these formulas makes it possible to investigate the bending vibrations of rods with dead weight taken into account under any boundary conditions. For example, in the work [11] the authors proposed new method of cantilever rod structures calculation with taking into account the dead weight.

Dynamic boundary conditions for the hinged rod have the form:

$$
y(0, t)=0 ; M(0, t)=0 ; y(l, t)=0 ; M(l, t)=0 .
$$

Realization of these conditions by exact formulas from [10] leads to the frequency equation, on the left side of which there appears a convergent series in powers of the dimensionless parameter $K$

$$
\eta_{0}+\eta_{1} K^{2}+\eta_{2} K^{4}+\eta_{3} K^{6}+\ldots=0
$$

The dimensionless coefficients of the equation $\eta_{k}(k=1,2,3, \ldots)$ are defined by the formulas

$$
\eta_{0}=1, \eta_{k}=\sum_{j=0}^{k}\left(\beta_{2, j}(l) \widehat{\beta}_{4, k-j}(l)-\widehat{\beta}_{2, j}(l) \beta_{4, k-j}(l)\right),
$$

where

$$
\begin{gathered}
\beta_{2,0}(l)=c_{2,0,0}+\sum_{j=1}^{\infty}(-1)^{j} \alpha^{j} c_{2,0, j}, \\
\beta_{4,0}(l)=c_{4,0,0}+\sum_{j=1}^{\infty}(-1)^{j} \alpha^{j} c_{4,0, j}, \\
\widehat{\beta}_{2,0}(l)=\left(f_{2,0,0}-1\right) f_{2,0,0} c_{2,0,0}+\sum_{j=1}^{\infty}(-1)^{j} \alpha^{j}\left(f_{2,0, j}-1\right) f_{2,0, j} c_{2,0, j}, \\
\widehat{\beta}_{4,0}(l)=\left(f_{4,0,0}-1\right) f_{4,0,0} c_{4,0,0}+\sum_{j=1}^{\infty}(-1)^{j} \alpha^{j}\left(f_{4,0, j}-1\right) f_{4,0, j} c_{4,0, j}, \\
\beta_{n, k}(l)=c_{n, k, 0}+\sum_{j=1}^{\infty}(-1)^{j} \alpha^{j} c_{n, k, j},
\end{gathered}
$$




$$
\begin{gathered}
\widehat{\beta}_{n, k}(l)=\left(f_{n, k, 0}-1\right) f_{n, k, 0} c_{n, k, 0}+\sum_{j=1}^{\infty}(-1)^{j} \alpha^{j}\left(f_{n, k, j}-1\right) f_{n, k, j} c_{n, k, j}, \\
\alpha=\frac{q l^{3}}{E I} .
\end{gathered}
$$

To determine the dimensionless coefficients of series (4)-(9), we use the set of recurrence formulas [10]:

$$
\begin{gathered}
c_{n, 0,0}=\frac{1}{(n-1) !} ; \\
c_{n, k, 0}=\frac{1}{(n+4 k-1) !} ; \\
c_{n, 0, j}=\frac{(n-1)(n+2) \ldots(n+3 j-4)}{(n+3 j-1) !} ; \\
c_{n, k, j}=\frac{c_{n, k-1, j}+\left(f_{n, k, j}-3\right)^{2} c_{n, k, j-1}}{\left(f_{n, k, j}-3\right)\left(f_{n, k, j}-2\right)\left(f_{n, k, j}-1\right) f_{n, k, j}},
\end{gathered}
$$

where

$$
f_{n, k, j}=n+4 k+3 j-1(n=2,4)(k=1,2,3, \ldots)(j=1,2,3, \ldots) .
$$

The dimensionless parameter $K$ will further be called the coefficient of vibrations.

Equations of the form (2) are often encountered in mechanics. To find solutions of such equations, the method of comparing the roots calculated for different numbers of retained terms of the series is used [12]. Such an approach makes it possible to calculate the roots of an equation with any given accuracy.

\subsection{Analytical formulas for the vibration frequencies}

In the publication [10] the formula was obtained for frequencies of free vibration of a rod with arbitrary boundary conditions:

$$
p_{j}=\frac{K_{j}}{l^{2}} \sqrt{\frac{E I}{m}}(j=1,2,3, \ldots),
$$

where $K_{1}, K_{2}, K_{3}, \ldots$-roots of the corresponding frequency equation. In our case, the frequency equation has the form (2). Consequently, the problem of determining the frequencies for a vertical hinged rod is reduced to finding dimensionless vibration coefficients from equation (2).

As can be seen from (2)-(9), the values of the roots $K_{1}, K_{2}, K_{3}, \ldots$ will depend on the dimensionless parameter $\alpha$. Let's set the actual range of this parameter change.

From the theory of stability, it is known $[13,14]$ that a hinged column under the influence of longitudinal distribution load with a value 


$$
q_{c r}=18.5687 \frac{E I}{l^{3}}
$$

will lose stability. Therefore, from the practical point of view it is meaningless to consider the problem of rod's vibrations for values of the parameter $q$ greater than $q_{c r}$. Assuming in formula (10) $q=q_{c r}$ and using (12), we find the maximum possible value of $\alpha$ :

$$
\alpha_{\max }=\frac{q_{c r} l^{3}}{E I}=18.5687
$$

Consequently, the values $\alpha$ relevant for the study lie in the interval

$$
0 \leq \alpha \leq 18.5687 \text {. }
$$

Table 1 shows the results of the calculation of the first three vibration coefficients for eleven values of the parameter $\alpha$ from the specified interval, equidistant from each other in steps 1.85687 .

Table 1. Vibration coefficients

\begin{tabular}{|c|c|c|c|}
\hline$\alpha$ & $K_{1}$ & $K_{2}$ & $K_{3}$ \\
\hline 0.0000 & 9.8696 & 39.4784 & 88.8264 \\
\hline 1.8569 & 10.2490 & 38.7144 & 88.4026 \\
\hline 3.7137 & 10.6561 & 37.9218 & 87.9758 \\
\hline 5.5706 & 11.0950 & 37.0967 & 87.5461 \\
\hline 7.4275 & 11.5708 & 36.2339 & 87.1133 \\
\hline 9.2844 & 12.0901 & 35.3270 & 86.6776 \\
\hline 11.1412 & 12.6617 & 34.3670 & 86.2388 \\
\hline 12.9981 & 13.2982 & 33.3416 & 85.7970 \\
\hline 14.8550 & 14.0173 & 32.2330 & 85.3520 \\
\hline 16.7118 & 14.8477 & 31.0126 & 84.9040 \\
\hline 18.5687 & 15.8392 & 29.6305 & 84.4528 \\
\hline
\end{tabular}

Using the data from Table 1, we represent the found correspondence between parameters $\alpha$ and $K$ in the analytical form. Considering the coefficient $K$ as a function of variable $\alpha$, and also having the set of values of this function, we approximate $K$ by a polynomial. To achieve high accuracy of approximation, the degree of the polynomial will be chosen from the condition that the coefficient of determination is not less than 0.9999 .

As calculations show, for the first and the second vibration coefficients the value of the determination coefficient will practically equal one for forth power approximation, and for the third - for a quadratic approximation. The final formulas have the form:

$$
K_{1}=\pi^{2}+0.189 \alpha+0.00659 \alpha^{2}-0.000291 \alpha^{3}+1.72 \cdot 10^{-5} \alpha^{4} ;
$$




$$
\begin{gathered}
K_{2}=4 \pi^{2}-0.3959 \alpha-0.00676 \alpha^{2}+0.00029 \alpha^{3}-1.7 \cdot 10^{-5} \alpha^{4} \\
K_{3}=9 \pi^{2}-0.22734 \alpha-0.00044 \alpha^{2}
\end{gathered}
$$

As a result, according to formulas (11), (14)-(16), to determine the first three frequencies of free vibrations of vertical hinged structures, we have the following analytical formulas:

$$
\begin{gathered}
p_{1}=\frac{\pi^{2}+0.189 \alpha+0.00659 \alpha^{2}-0.000291 \alpha^{3}+1.72 \cdot 10^{-5} \alpha^{4}}{l^{2}} \sqrt{\frac{E I}{m}} \\
p_{2}=\frac{4 \pi^{2}-0.3959 \alpha-0.00676 \alpha^{2}+0.00029 \alpha^{3}-1.7 \cdot 10^{-5} \alpha^{4}}{l^{2}} \sqrt{\frac{E I}{m}} \\
p_{3}=\frac{9 \pi^{2}-0.22734 \alpha-0.00044 \alpha^{2}}{l^{2}} \sqrt{\frac{E I}{m}} .
\end{gathered}
$$

Using formulas (10), (17)-(19), knowing the characteristics of $q, l, E I$, it is possible in the future to calculate the vibration frequencies of hinged structures with dead weight taken into account without using approximate methods and program complexes.

The vibrations frequencies for a weightless hinged rod are given by the known formulas $[15,16]$ :

$$
\begin{aligned}
& \omega_{1}=\frac{\pi^{2}}{l^{2}} \sqrt{\frac{E I}{m}} ; \\
& \omega_{2}=\frac{4 \pi^{2}}{l^{2}} \sqrt{\frac{E I}{m}} ; \\
& \omega_{3}=\frac{9 \pi^{2}}{l^{2}} \sqrt{\frac{E I}{m}} .
\end{aligned}
$$

It is obvious that formulas (20)-(22) are a special case of formulas (17)-(19).

With the help of (17)-(22), it is easy to establish a connection between the frequencies taking into account the dead weight and frequencies without taking into account the dead weight of the rod:

$$
\begin{gathered}
\frac{p_{1}}{\omega_{1}}=1+0.0191 \alpha+0.000668 \alpha^{2}-2.95 \cdot 10^{-5} \alpha^{3}+1.74 \cdot 10^{-6} \alpha^{4} ; \\
\frac{p_{2}}{\omega_{2}}=1-0.010 \alpha-0.000171 \alpha^{2}+7.35 \cdot 10^{-6} \alpha^{3}-4.31 \cdot 10^{-7} \alpha^{4} ; \\
\frac{p_{3}}{\omega_{3}}=1-0.00256 \alpha-4.95 \cdot 10^{-6} \alpha^{2} .
\end{gathered}
$$

Based on (13), (23)-(25), we can also establish the change limits for the frequency ratios: 


$$
\begin{aligned}
& 1 \leq \frac{p_{1}}{\omega_{1}}<1.61 ; \\
& 0.75<\frac{p_{2}}{\omega_{2}} \leq 1 ; \\
& 0.95<\frac{p_{3}}{\omega_{3}} \leq 1 .
\end{aligned}
$$

Formula (10) with taking into account (12) can be rewritten in the form

$$
\alpha=18.5687 \frac{q}{q_{c r}}
$$

Substituting the value (29) instead of parameter $\alpha$ in formulas (23)-(25), we express the frequency ratios in terms of the distributed longitudinal load $q$ :

$$
\begin{gathered}
\frac{p_{1}}{\omega_{1}}=1+0.3556 \frac{q}{q_{c r}}+0.2302\left(\frac{q}{q_{c r}}\right)^{2}-0.1887\left(\frac{q}{q_{c r}}\right)^{3}+0.2071\left(\frac{q}{q_{c r}}\right)^{4} \\
\frac{p_{2}}{\omega_{2}}=1-0.1862 \frac{q}{q_{c r}}-0.059\left(\frac{q}{q_{c r}}\right)^{2}+0.0470\left(\frac{q}{q_{c r}}\right)^{3}-0.0512\left(\frac{q}{q_{c r}}\right)^{4} \\
\frac{p_{3}}{\omega_{3}}=1-0.04752 \frac{q}{q_{c r}}-0.0017\left(\frac{q}{q_{c r}}\right)^{2} .
\end{gathered}
$$

Fig. 3-5 show the graphical dependence of the frequency ratios on parameter $q$.

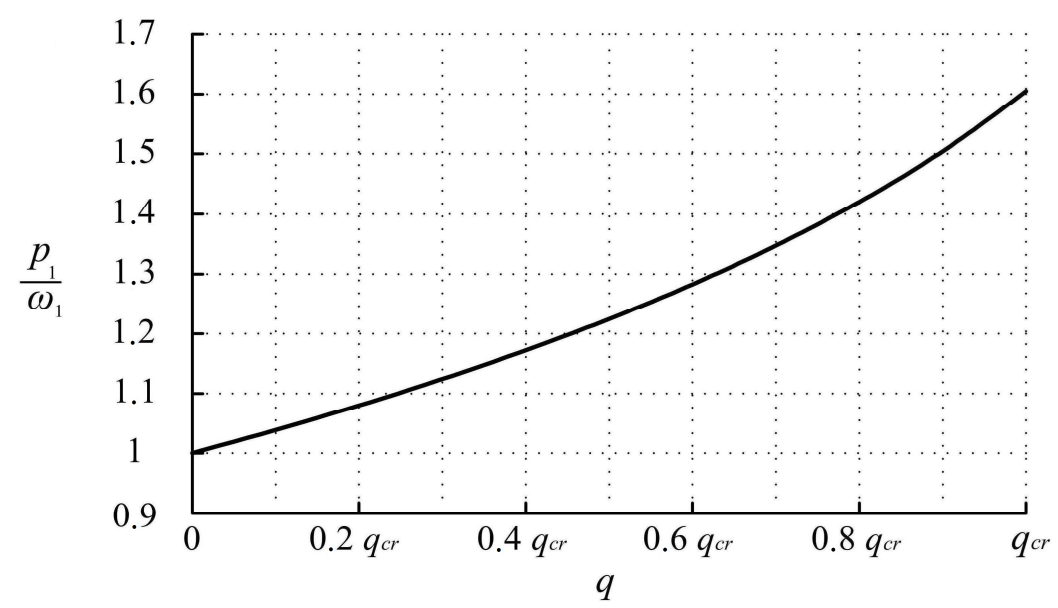

Fig. 3. Graph of the dependence of $p_{1} / \omega_{1}$ on the longitudinal load $q$. 


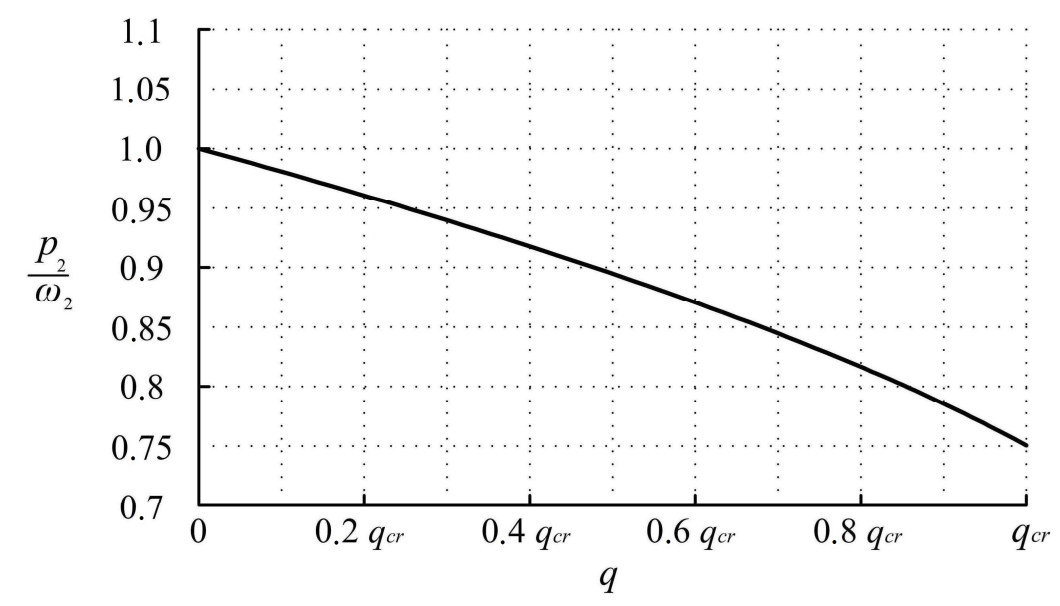

Fig. 4. Graph of the dependence of $p_{2} / \omega_{2}$ on the longitudinal load $q$.

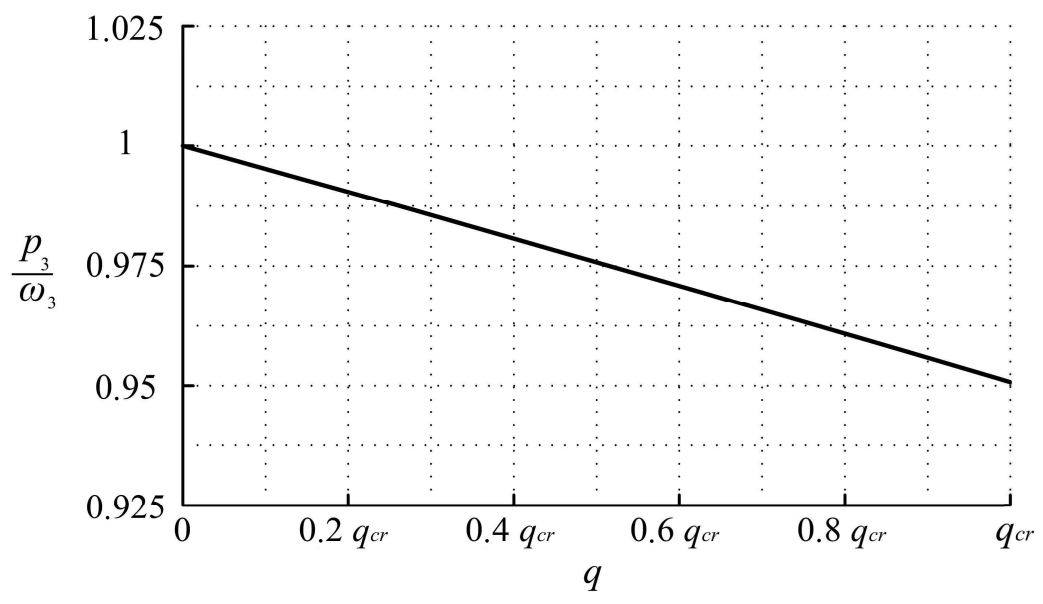

Fig. 5. Graph of the dependence of $p_{3} / \omega_{3}$ on the longitudinal load $q$.

As can be seen from the graphs, taking into account the rod's dead weight leads to an increase the first frequency and a decrease the second and the third one. The greater the value of the longitudinal load, the greater the difference between the frequencies of a weighty rod and the frequencies of a weightless rod.

\section{Conclusions}

Based on the exact solution of the differential equation of vibrations of a weighted uniform rod, the following results for hinged vertical structures with dead weight taken into account are obtained:

1. Formulas for the first three frequencies of vibrations (17)-(19) are found in an analytical form. Using these formulas, it is possible to calculate frequencies without using approximate methods and program complexes.

2. The dependence between the frequencies with taking into account the dead weight of structure and without it, was established analytically (23)-(25). It was established that taking into account dead weight leads to an increase in the first frequency and a decrease in 
the second and the third frequency, in comparison with the corresponding frequencies for the weightless rod.

3. The greater the value of the longitudinal load, the greater the difference between the frequencies of the weighty rod and the frequencies for the weightless rod. From the formulas (26)-(28) we come to the following conclusions:

- the difference between $p_{1}$ and $\omega_{1}$ does not exceed $61 \%$;

- the difference between $p_{2}$ and $\omega_{2}$ does not exceed $25 \%$;

- the difference between $p_{3}$ and $\omega_{3}$ does not exceed $5 \%$.

The maximum difference will be achieved at a value of $q=q_{c r}$.

4. Formulas (30)-(32) established an analytical relationship $p_{j} / \omega_{j}(j=1,2,3)$ between the frequencies when the longitudinal load value changes.

In the future, based on the results of the publication [10], similar studies can be carried out for structures under the other boundary conditions.

\section{References}

1. R. Luo, Journal of Mechanical Engineering Science, 228 (1) (2013)

2. S. Naguleswaran, International Journal of Mechanical Engineering Education, 33 (1) (2005)

3. E. Ghandi, B. Rafezy, Journal of Structural and Construction engineering, 84 (2016).

4. S. Azhermachev, Construction and industrial safety, 33-34 (2010)

5. S. S. Rao, Mechanical vibrations (Pearson, Prentice Hall, 2004)

6. F. J. Shaker, Lewis Research Center Report NASA TN D-8109 (December 1975)

7. A. Bokaian, Journal of Sound and Vibration, 126 (1) (1998)

8. M. Vasilenko, A. Alekseychuk, Teoriya kolyvan' ta stiykosti rukhu (Kyiv, High School, 2004)

9. E. Khachiyan, Seysmicheskoye vozdeystvie na vysotnyie zdaniya $i$ sooruzheniya (Ayastan, 1973)

10. Yu. Krutii, M. Suriyaninov, V. Vandynskyi, MATEC Web of Conferences, 116 (2017)

11. Yu. Krutii, M. Suriyaninov, V. Vandynskyi, Eastern-European Journal of Enterprise Technologies, 3/7 (93) (2018)

12. V. P. Ilyin, A. M. Karpov, A. M. Maslennikova, Chislennye metody resheniya zadach stroitelnoy mekhaniki (Vysheyshaya shkola, 1990)

13. C. M. Wang, C. Y. Wang, J. N. Reddy, Exact solutions for buckling of structural members (CRC Press, 2005)

14. N. S. Volmir, Ustoychivost uprugih sistem (Fizmatgiz, 1963)

15. V. A. Bazhenov, A. V. Perelmuter, O. V. Shishov, Budivel'na mekhanika. Kompyuterni tekhnolohii (Kyiv, Karavela, 2009)

16. V. A. Kiseliov, Stroitelnaya mekhanika (Moscow, Stroyizdat, 1980) 